\title{
Delayed diagnosis of Birt-Hogg-Dubé syndrome due to marked intrafamilial clinical variability: a case report
}

\author{
E. C. Sattler ${ }^{1 *}$ and O. K. Steinlein ${ }^{2}$
}

\begin{abstract}
Background: Birt-Hogg-Dubé syndrome is a genetic syndrome caused by mutations in the FLCN gene. The main symptoms are lung bullae and pneumothorax, benign and malignant kidney tumors, and facial fibrofolliculoma. The risk of pneumothorax is considerable between ages 20-40 years, but decreases markedly after this age range and first-time pneumothorax after age 50 years is rare. Fibrofolliculomas usually occur between ages 35 and 45 years, while the risk for kidney cancer increases steadily with age, starting in young adulthood. However, we demonstrate here that within the same family patients might develop symptoms significantly before or after the usual age range, obscuring the typical clinical pattern and delaying diagnosis.

Case presentation: The 43 year old index patient had a history of lung bullae and recurrent pneumothoraces starting 14 years earlier. His father (age 83 years) and one of the paternal uncles experienced their first pneumothorax unusually late after the age of 60 years. The uncle subsequently had four more pneumothoraces, and was diagnosed with kidney in his early 70s. Considerable differences in age of onset were also observed with regard to facial fibrofolliculomas that both paternal uncles developed very early around age 20 years, but which the father only started to show in his eighth decade. Birt-Hogg-Dubé syndrome was finally diagnosed when the index patient started to develop fibrofolliculomas within the typical age range.

Conclusions: The family described here illustrates that Birt-Hogg-Dubé syndrome can be difficult to recognize, if presenting with considerable intrafamilial clinical variability. With a life-time kidney cancer risk of about 14-35\% the consequences of delayed diagnosis might be grave for the affected family members. The possibility of Birt-Hogg-Dubé syndrome should therefore be taken into consideration in apparently sporadic patients presenting with lung bullae and pneumothorax.
\end{abstract}

Keywords: Birt-Hogg-Dubé syndrom, FLCN, Pneumothorax, Lung bullae, Kidney cancer

\section{Background}

Birt-Hogg-Dubé syndrome (BHDS) is a genetic cancer syndrome characterized by fibrofolliculomas, lung bullae with the risk of spontaneous pneumothorax, and benign or malignant kidney tumors. The syndrome is caused by mostly truncating mutations in the FLCN gene that encodes folliculin, a putative tumor suppressor protein [1]. The clinical expression shows inter- and intrafamilial variability, but the onset of symptoms usually follows an age-dependent pattern. Lung cysts are commonly found

\footnotetext{
* Correspondence: Elke.Sattler@med.uni-muenchen.de

1 Department of Dermatology and Allergology, University Hospital, Ludwig

Maximilian University of Munich, Munich, Germany

Full list of author information is available at the end of the article
}

(in $85 \%$ of patients) and peumothoraces (often recurrent) affect about 25-30\% of patients [2]. After age 50 years peumothoraces are rather rare in BHDS patients. Starting in young adulthood, the risk for renal cancer steadily increases with age, leading to an average cumulative life time risk of $14-35 \%$ [3-5]. The majority of patients (about 80\%) develop fibrofolliculomas which are mostly located on the face but can also be found on the neck and the upper trunk [6-8]. In most families the autosomal mode of inheritance is obvious, because the onset of symptoms between young adulthood and middle age ensures that patients have personal knowledge of affected relatives in one or more previous generations. The BHDS family we describe here demonstrates that 
the mode of inheritance, and therefore the cancer risk, might be obscured by family members presenting with an unusual late onset of symptoms.

\section{Case presentation}

The index patient (III6, Fig. 1) was referred to our BHD interdisciplinary outpatient clinic at age 43 years, after he had suffered from more than five pneumothoraces caused by rupture of lung bullae starting at age 29 years (Fig. 2). Facial fibrofolliculoma began to occur at the age of 41 years. Results of screening examinations including computer tomography of kidneys, colonoscopy and ultrasonography of the thyroid gland were normal. The family's medical history was obtained from the index patient. His only son (IV1), 15 years of age, is reportedly healthy, but - in agreement with German law - hasn't been genetically tested yet. The index patient's father (age 83 years) (II9) had his first and so far only pneumothorax at age 61 years. He noticed facial fibrofolliculomas for the first time 2-3 years ago around age 80 years. He suffers from chronic renal insufficiency stage III but has no known kidney tumors. Two of the father's brothers (II4, II7) reportedly have multiple facial fibrofolliculomas since age 20 years. One of them (II7) had five spontaneous pneumothoraces starting after age 60 years and was diagnosed with kidney cancer and prostate cancer in his early 70s. The father also has four siblings (II1-2, II5, II8) not showing any BHDS symptoms. It is unknown, if pneumothorax or facial fibrofolliculomas occurred in the grandparents generation. The medical history of the index patient's maternal family (II10) was unremarkable. Both sides of the family are of German origin. The CARE guidelines were followed in reporting this case.

Mutation screening of the FLCN complete coding region and adjacent intronic sequences was performed by PCR and subsequent Sanger sequencing following standard protocols. For each PCR 50-100 ng DNA were amplified using the AmpliTAq Gold kit (Thermo Fisher

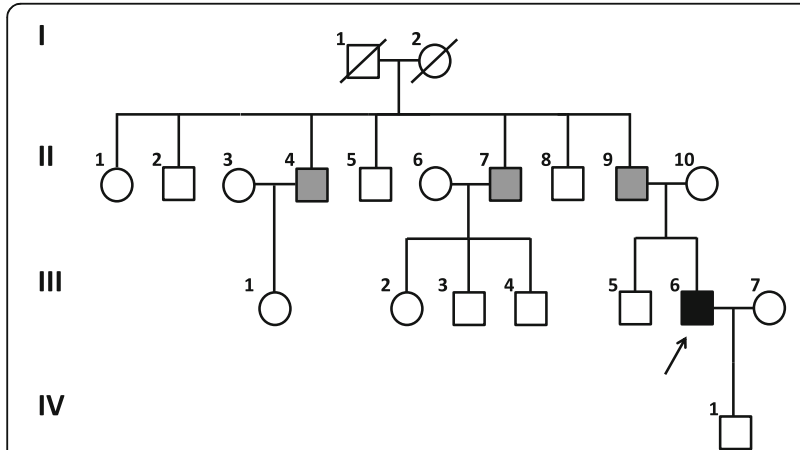

Fig. 1 Pedigree of BDHS family. The index patient is marked by an arrow. Black symbol, BHDS confirmed by mutation analysis; grey symbols, clinical suspicion of BHDS

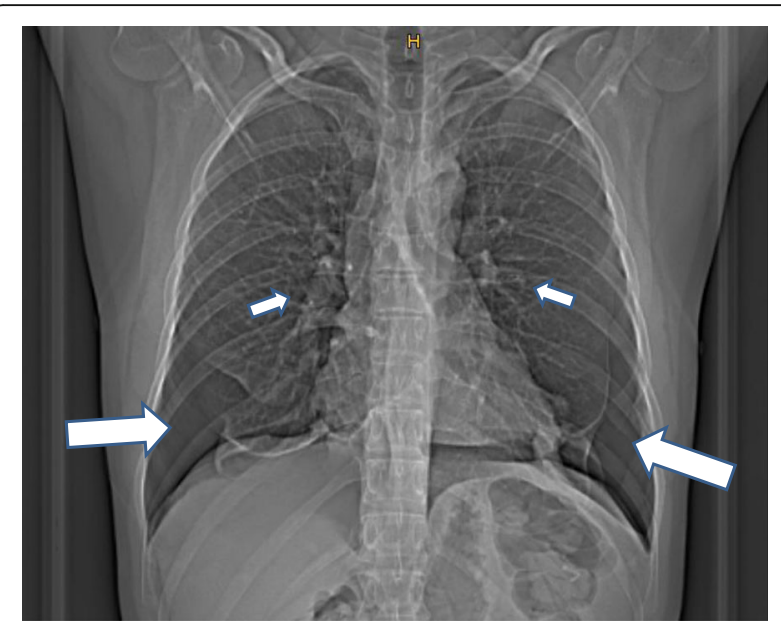

Fig. 2 Chest computed tomography scan from the index patient. Bilateral pneumothorax (large arrows) and lung bullae (small arrows) are indicated. The pneumothorax is mostly localized in the basal parts of the chest cavity due to previous pleurodesis treatment

Scientific, Dreieich, Germany) and the amplification products were subsequently purified by Quiagen PCR purification kit (Quiagen, Hilden, Germany). Amplicons were sequenced with the 3500 Genetic Analyser (Thermo Fisher Scientific, Dreieich, Germany). Sequencing of the FLCN gene in the index patient showed heterozygosity for the mutation c.1285dupC (p.His429ProfsX27) within exon 11 (Fig. 3).

\section{Discussion and conclusions}

The index patient has been suffering from recurrent pneumothorax for 14 years before the diagnosis of BHDS was made. This delay most likely had different reasons, mainly due to the the fact that BHDS is still one of the lesser known inherited syndromes. However, the untypical family history certainly contributed to the delay, in particular the unusually late manifestation of pneumothorax in the paternal generation. Pneumothorax is often the first clinically recognized symptom in BHDS patients, typically occurring between age 20 and 40 years $[3,6]$. The likeliness for pneumothorax in BHDS patients markedly decreases in the second half of life. A first-time pneumothorax in the sixth decade, as happened twice in the family presented here (II7, II9), has rarely been reported in BHDS patients [9]. Another factor contributing to the delay in diagnosis was the atypical age of onset regarding fibrofolliculomas. These benign skin tumors mostly start to appear between the ages 35 and 45 years, often slowly but steadily increasing in numbers during subsequent years $[6,10]$. In both paternal uncles (II4, II7) these benign skin tumors occurred at the unusually early age of 20 years, while the index patient's father only started to develop them at a very advanced age. Taken together, affected family 


\section{c.1285dupC}

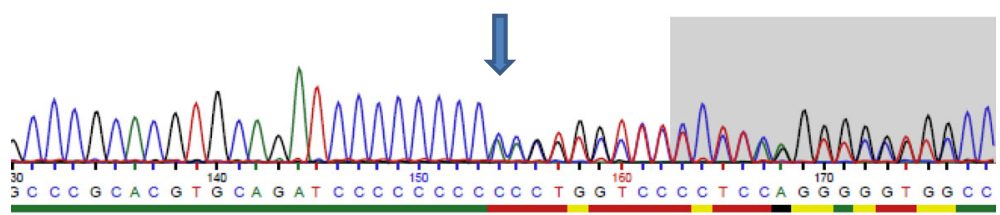

Fig. 3 Sequence electropherogram demonstrating the mutation c.1285dupC (p.His429ProfsX27) within exon 11. The position of the duplicated cytosine is marked by an arrow

members showed a considerable variation in both age of onset and clinical course, rendering it particularly difficult to recognize the inheritability of the condition. The possibility of BHDS was only taken into consideration, when the index patient started to develop fibrofolliculomas at a typical age. As a consequence of the delay in diagnosis the affected family members remained unaware of their high or elevated tumor risk and their need for regular kidney cancer screening for many decades.

There is no evidence that the intrafamilial variation demonstrated by the index patient and his relatives can be explained by genotype-phenotype correlation. The mutation found in our family, c.1285dupC, is located in a mutational hotspot in exon 11 and accounts for 25-44\% of all FLCN mutations [11-13]. Patients with this mutation therefore had a major impact on published clinical descriptions from which the above cited typical onset ages of BHD symptoms were taken. The family described here represents an example of the wide age range at which clinical manifestations can occur. It is likely that the clinical course is modulated by other, unknown genetic or non-genetic factors. The identification of such modifying factors is a challenge that will require further recruitment of BHDS families presenting with unusual clinical histories.

The inherited syndrome BHDS can be difficult to diagnose, if presenting with significant intrafamilial clinical heterogeneity. The family described here demonstrates that monosymptomatic expression or manifestation of symptoms far outside the typical age range might delay diagnosis. Considering the cumulative life time risk of $14-35 \%$ for kidney tumors the consequences of such a delay are potentially serious for the affected family members. As demonstrated by Park et al. [13], the diagnosis of BHDS should always be taken into consideration in apparently healthy younger adults presenting with a combination of atypically localized lung bullae and pneumothorax, even in the absence of a typical family history.

\section{Acknowledgements}

We wish to thank the index patient for participating in this study and for his valuable help in drafting this manuscript.

\section{Funding}

This study was supported by the Verein zur Förderung von Wissenschaft und Forschung an der Medizinischen Fakultät der Ludwig-Maximilians-Universität München e. V.

Availability of data and materials

All data generated or analysed during this study are included in this published article.

\section{Authors' contributions}

OS and ES wrote the manuscript, OS carried out the genetic counselling and participated in molecular genetic studies, ES performed the clinical examinations. Both authors and the index patient revised and approved the final version of this manuscript.

\section{Ethics approval and consent to participate}

All research was conducted according to the declaration of Helsinki principles. The study has been approved of by the ethical committee/ institutional review board (IRB) of the Medical Faculty, University Hospital Munich, under the project-number: 508/16UE.

\section{Consent for publication}

Written informed consent for the publication of medical data (including those of the index patients son), images, and medical history of family members was obtained from the index patient.

\section{Competing interests}

The authors declare that they have no competing interests.

\section{Publisher's Note}

Springer Nature remains neutral with regard to jurisdictional claims in published maps and institutional affiliations.

\section{Author details}

'Department of Dermatology and Allergology, University Hospital, Ludwig Maximilian University of Munich, Munich, Germany. ${ }^{2}$ Institute of Human Genetics, University Hospital, Ludwig Maximilian University of Munich, Goethestr. 29, D-80336 Munich, Germany.

Received: 2 June 2017 Accepted: 8 March 2018

Published online: 16 March 2018

References

1. Baba M, Hong SB, Sharma N, Warren MB, Nickerson ML, Iwamatsu A, et al. Folliculin encoded by the BHD gene interacts with a binding protein, FNIP1, and AMPK, and is involved in AMPK and mTOR signaling. Proc Natl Acad Sci U S A. 2006:103:15552-7.

2. Zbar B, Alvord WG, Glenn G, Turner M, Pavlovich CP, Schmidt L, et al. Risk of renal and colonic neoplasms and spontaneous pneumothorax in the BirtHogg-Dubé syndrome. Cancer Epidemiol Biomark Prev. 2002;11:393-400.

3. Houweling AC, Gijezen LM, Jonker MA, van Doorn MB, Oldenburg RA, van Spaendonck-Zwarts KY, et al. Renal cancer and pneumothorax risk in Birt- 
Hogg-Dubé syndrome; an analysis of 115 FLCN mutation carriers from 35 BHD families. Br J Cancer. 2011;105:1912-9.

4. Toro JR, Wei MH, Glenn GM, Weinreich M, Toure O, Vocke C, et al. BHD mutations, clinical and molecular genetic investigations of Birt-Hogg-Dubé syndrome: a new series of 50 families and a review of published reports. J Med Genet. 2008:45:321-31.

5. Nickerson ML, Warren MB, Toro JR, Matrosova V, Glenn G, Turner ML, et al. Mutations in a novel gene lead to kidney tumors, lung wall defects, and benign tumors of the hair follicle in patients with the Birt-Hogg-Dubé syndrome. Cancer Cell. 2002;2:57-64.

6. Menko FH, van Steensel MA, Giraud S, Friis-Hansen L, Richard S, Ungari S, et al. Birt-Hogg-Dube syndrome: diagnosis and management. Lancet Oncol. 2009;10:1199-206.

7. Schmidt LS, Linehan WM. Molecular genetics and clinical features of BirtHogg-Dubé syndrome. Nat Rev Urol. 2015;12:558-69.

8. Khoo SK, Giraud S, Kahnoski K, Chen J, Motorna O, Nickolov R, et al. Clinical and genetic studies of Birt-Hogg-Dube syndrome. J Med Genet. 2002;39(12):906.

9. Gupta N, Kopras EJ, Henske EP, James LE, El-Chemaly S, Veeraraghavan S, et al. Spontaneous Pneumothoraces in patients with Birt-Hogg-Dubé syndrome. Ann Am Thorac Soc. 2017; https://doi.org/10.1513/AnnalsATS.201611-8860C

10. Vernooij M, Claessens T, Luijten M, van Steensel MA, Coull BJ. Birt-HoggDubé syndrome and the skin. Familial Cancer. 2013;12:381-5.

11. Lim DH, Rehal PK, Nahorski MS, Macdonald F, Claessens T, Van Geel M, et al. A new locus-specific database (LSDB) for mutations in the folliculin (FLCN) gene. Hum Mutat. 2010;31:E1043-51.

12. Wei MH, Blake PW, Shevchenko J, Toro JR. The folliculin mutation database: an online database of mutations associated with Birt-Hogg-Dubé syndrome. Hum Mutat. 2009;30:E880-90.

13. Park HJ, Park CH, Lee SE, Lee GD, Byun MK, Lee S. Birt-Hogg-Dube syndrome prospectively detected by review of chest computed tomography scans. PLoS One. 2017;12:e0170713.

\section{Submit your next manuscript to BioMed Central and we will help you at every step:}

- We accept pre-submission inquiries

- Our selector tool helps you to find the most relevant journal

- We provide round the clock customer support

- Convenient online submission

- Thorough peer review

- Inclusion in PubMed and all major indexing services

- Maximum visibility for your research

Submit your manuscript at www.biomedcentral.com/submit

) Biomed Central 